\title{
Generating work from macro cavities in superheated liquids
}

\author{
P. A. F. Pereira ${ }^{1}$, H. E. Schulz ${ }^{1,2}$ \& G. Lombardi ${ }^{1}$ \\ ${ }^{1}$ Nucleus of Thermal Sciences and Engineering, \\ University of São Paulo, Brazil \\ ${ }^{2}$ Department of Hydraulics and Sanitary Engineering, \\ University of São Paulo, Brazil
}

\begin{abstract}
Considering liquid phases, it is well known that pure substances (or almost pure) can be maintained in the liquid phase for temperatures much higher than those of the liquid-vapor phase change. In these superheated cases, it is necessary to startup the phase change through some adequate mechanism, generating a very fast sequence of phenomena. In the present study, a confined liquid (water) was subjected to an adequate situation of pressure and temperature that allowed macro cavities to be formed, where sudden expansion generated a piston-like movement while the liquid dislocated. The geometrical arrangements of the equipment allow the "use" of the reaction force, by stretching a spring. The aim of this study was to analyse the evolution of macro cavities generated by heating water under low pressure, describing the details of their dynamics and work production. First tests were performed using distilled water and a glass container (an adequately prepared mercury lamp) in which the generation and collapse of cavities with a diameter of about $10^{-1} \mathrm{~m}$ were observed, and peak forces of 400 to $500 \mathrm{~N}$ were measured. A one-dimensional governing equation was obtained from the usual conservation equations, and the results show that it considers the relevant aspects for the quantification of the observed movements. A second experimental system, that allows a better approximation to the one-dimension movement, is also presented. The results show that the sudden expansions can be used to generate work (although a small quantity), which may be of interest in situations where energy, like solar energy, can be freely obtained from the environment.
\end{abstract}

Keywords: macro-scale cavities, bubble dynamics, ebullition, usable cavitation. 


\section{Introduction}

Cavitation is the phenomenon of generation, growing and collapsing of lowpressure bubbles (cavities) in a liquid. This phenomenon may involve high amounts of energy, which interaction with the surrounding liquid or boundaries may be viewed as positive or negative, depending if the consequences are “desired” or not. Falvey [1], Sánchez et al. [2], and d’Agostino and Salvetti [3] describe intense erosion events caused by cavitation in hydraulic structures, corrosion effects in propellers and turbines, vibrations, losses of efficiency, noise, which are undesired aspects of cavitation in mechanical devices. Kodama and Tomita [4] mention negative effects in photomedicine, when using pulsed lasers in angioplasty, orthopedic surgeries, dentistry and ophthalmology. Energy can be absorbed by the tissue, generating cavitation bubbles and shock waves, damaging the tissue.

Considering the positive aspects, Alehossein and Qin [5] describe cavitation water jets used to cut and drill rocks in the mining industry, while Azuma et al. [6] and Assis et al. [7] mention the use of cavitation as a physical disinfectant of contaminated waters, in which high-pressure waves and high-velocity microjets may eliminate microorganisms present in wastewaters. Perhaps the most intriguing phenomenon related to cavitation is the sonoluminescence, that is, the light that emanates from fluids at rest and excited by sound waves, as described, for example, by Gaitan et al. [8], Brenner et al. [9], and Wrbanek et al. [10]. In this sense, the present study considers the possibility of generating usable work from macro-cavities in fluids, that is, a positive aspect of cavitation.

Following Tullis [11], cavitation and ebullition are similar events generated when following different ways. Cavitation is attained by lowering the pressure of a liquid at constant temperature, while ebullition is attained by increasing the temperature of a liquid at constant pressure. Independently of the procedure, cavities are formed when pressure and temperature attain the vapor condition.

Different points of view were followed in the study of cavitation. A review explaining some main concepts and equations on cavitation and bubble dynamics was furnished by Shima [12]. The author discusses, for example, equations for the bubble nuclei, effects of compressibility, effects of solid walls, and characteristics of the Shima-Tomita approximation. Perhaps the most known equation for cavitation is that of the bubble surface velocity in an infinite liquid, as presented by Rayleigh in 1917, Plesset in 1949, and Poritsky in 1952 (see Leighton [13]). It may be said that these equations consider the movement of the fluid that surrounds the bubble, and the work done by the displaced liquid may be calculated by the product of the ambient pressure and the maximum volume of the bubble, as shown by Tomita [14], for example. In the mentioned studies the results and the images obtained from cavitation experiments involve bubbles with size of several millimeters.

The diameters of the bubbles considered in the present study are more than 10 times bigger than those of the mentioned studies (of the order of $10 \mathrm{~cm}$ ). The volumes are, thus, more than 1000 times bigger, which implies in a huge gas "generation" (conversion of liquid into gas). Additionally, it was intended to 
obtain a main one-dimensional movement, so that a one-dimensional equation was proposed. Further, the movement of the solid wall which is in contact with the liquid is the relevant parameter, and not the movement of the liquid itself. In this case, the obtained equation considers the movement of the solid container. Although involving bigger dimensions, the growing and collapsing of bubbles occurred in a time interval of about only 60 milliseconds. The cavities were generated by locally heating water at a constant and low pressure. In this case, they were "ebullition events".

The aim of this study was 1) to produce a movement similar to a moving piston using macro-scale cavities and to verify its ability to realize work by stretching a spring; 2) to quantify the movement by using approximations that linearize a proposed governing differential equation.

Images obtained from the experiments involving the formation of the cavities and different correlated phenomena are also presented.

\section{Quantifying the one-dimensional movement}

The sequence of steps presented here are based on the study of Schulz et al. [15]. Consider the scheme of the container with liquid of fig. 1(a).
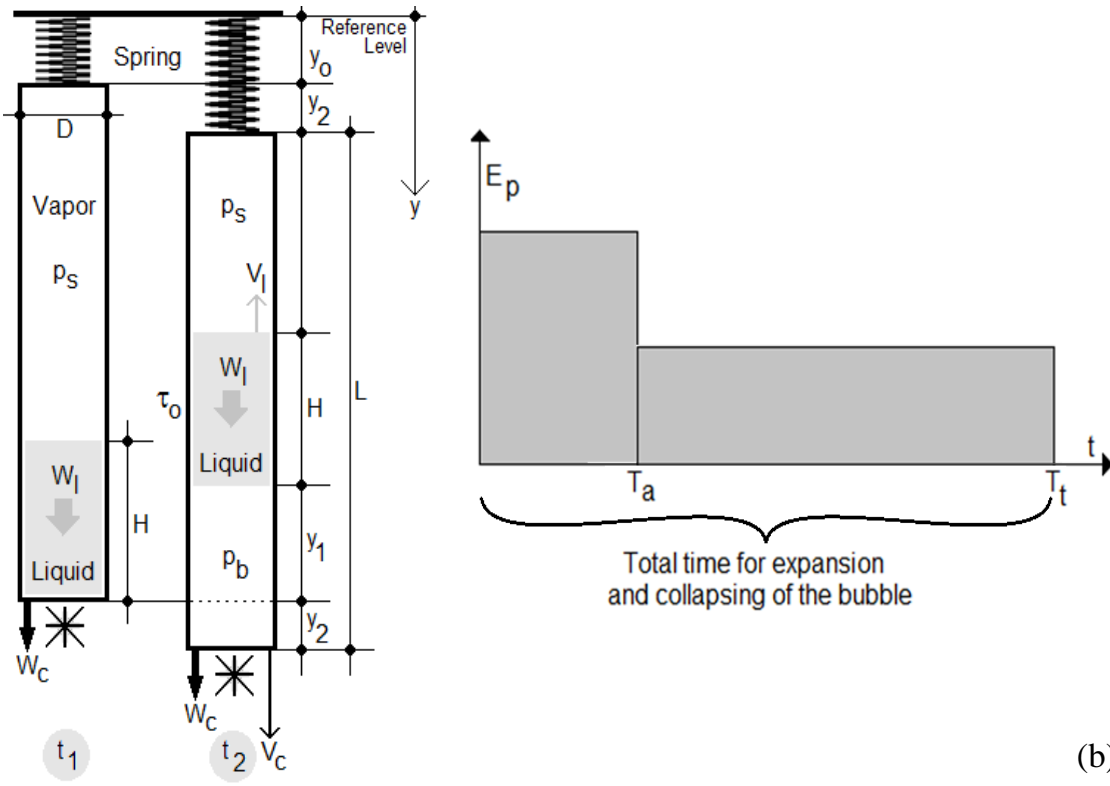

(a)

Figure 1: (a) Container with liquid. The star at the bottom indicates a heat source (heat). $t_{1}$ represents a time before the cavity has formed. $t_{2}$ represents a time after the cavity was formed. (b) The simplest evolution of the excess of pressure $\left(E_{p}\right)$ in the lower atmosphere $\left(p_{b}\right)$, assumed constant in two steps. 
In fig. 1(a), $\mathrm{D}$ is the diameter of the container, $\mathrm{m} ; \mathrm{H}$ is the thickness of the liquid phase, $\mathrm{m}$; $\mathrm{p}_{\mathrm{b}}$ is the pressure of the gaseous phase close to the bottom, $\mathrm{Pa}$; $\mathrm{p}_{\mathrm{s}}$ is the pressure of the gaseous phase above the liquid, $\mathrm{Pa}$; $\mathrm{t}$ is the time, $\mathrm{s} ; \mathrm{V}_{\mathrm{c}}$ is the velocity of the container (relative to the observer), $\mathrm{m} / \mathrm{s} ; \mathrm{V}_{\mathrm{l}}$ is the upwards velocity, $\mathrm{m} / \mathrm{s} ; \mathrm{W}_{\mathrm{c}}$ is the weight of the container, $\mathrm{N} ; \mathrm{W}_{\mathrm{l}}$ is the liquid weight, $\mathrm{N}, \mathrm{y}_{0}$ is the initial displacement of the spring, $\mathrm{m}$; $\mathrm{y}_{1}$ is the displacement of the liquid, $\mathrm{m} ; \mathrm{y}_{2}$ is the additional displacement induced by the cavity, $\mathrm{m} ; \tau_{0}$ is the shear stress between the container and the liquid, $\mathrm{Pa}$. In fig. 1(b), $\mathrm{E}_{\mathrm{p}}$ is the excess of pressure, $\mathrm{Pa}$.

The cylindrical container and the liquid are initially at rest $\left(\mathrm{t}_{1}\right)$, in thermal equilibrium with the surroundings, and fixed to the spring. The geometry allows a piston-like movement. The cavity (bubble) formed at the bottom of the container (by exchanging heat with the heat source) dislocates the liquid upwards $\left(\mathrm{t}_{2}\right)$. As a reaction, the container moves downwards.

From fig. 1(a), a bubble is formed only if $p_{b}$ obeys $p_{b}>W_{l} / A+p_{s}$, or

$$
p_{b}=\frac{4 W_{l}}{\pi D^{2}}+p_{s}+E_{p}
$$
by:

$E_{p}$ is an "excess of pressure". The resultant force on the liquid, $R_{\text {liq }}$, is given

$$
R_{\text {liq }}=-\left(p_{b}-p_{s}\right) \frac{\pi D^{2}}{4}+\tau_{0} \pi D H+W_{l}=-E_{p} \frac{\pi D^{2}}{4}+\tau_{0} \pi D H .
$$

$\tau_{0}$ is the shear stress indicated in fig. 1(a). For $t_{2}$, the resultant force on the inner side of the container is:

$$
R_{\text {inner }}=\left[\left(p_{b}-p_{s}\right) \frac{\pi D^{2}}{4}-\tau_{0} \pi D H\right]=W_{l}+\left[\frac{E p \pi D^{2}}{4}-\tau_{0} \pi D H\right] .
$$

The "external" forces are the weight of the container, $W_{c}$, the force of the spring, and the sum of resistance forces, $F_{R}$. The resultant is:

$$
R_{\text {outer }}=W_{c}-K\left(y_{0}+y_{2}\right) \mp F_{R} \text {. }
$$

$K$ is the constant of the spring, $\mathrm{N} / \mathrm{m}, y_{0}$ is its initial displacement, and $y_{2}$ is the additional displacement induced by the cavity. $F_{R}$ changes its sign for upwards and downwards movements. The resultant force on the container, $R_{c o n}$, is the sum of eqns (3) and (4):

$$
R_{\text {con }}=W_{c}+W_{l}-K\left(y_{0}+y_{2}\right)+\left[\frac{E_{p} \pi D^{2}}{4}-\tau_{0} \pi D H\right] \mp F_{R} .
$$

For $t_{1}$, system at rest, we have:

$$
W_{c}+W_{l}-K y_{0}=0 \text {. }
$$


From eqns (2), (5), and (6), the following set of two equations is obtained:

$$
m_{\text {con }} \frac{d V_{c}}{d t}=-K y_{2}+\left[\frac{E_{p} \pi D^{2}}{4}-\tau_{0} \pi D H\right] \mp F_{R}, \quad m_{\text {liq }} \frac{d V_{l}}{d t}=-E_{p} \frac{\pi D^{2}}{4}+\tau_{0} \pi D H .
$$

Eqn (7) are coupled through $\tau_{0}$ and $E_{p}$. If both variables are functions only of time, eqn (7) may be viewed as independent linear second order differential equations for $y_{1}$ and $y_{2}$. Assuming the resistance forces as proportional to the velocities (Bronson and Costa, for oscillatory problems [17]). $\tau_{0}=\alpha\left(V_{l}+V_{c}\right)$ and $F_{R}=a d y_{2} / d t$, where $\alpha$ and $a$ are proportionality coefficients. Eqn (7) can then be combined, furnishing:

$$
\frac{d^{3} y_{2}}{d t^{3}}+\left[\frac{a+\alpha \pi D H}{m_{\text {con }}}+\frac{\alpha \pi D H}{m_{\text {liq }}}\right] \frac{d^{2} y_{2}}{d t^{2}}+\left[\frac{K}{m_{\text {con }}}+\frac{\alpha \pi a D H}{m_{\text {con }} m_{\text {liq }}}\right] \frac{d y_{2}}{d t}+\frac{K \alpha \pi D H}{m_{\text {con }} m_{\text {liq }}} y_{2}=\frac{\pi D^{2}}{4 m_{\text {con }}} \frac{d E_{p}}{d t} .
$$

Of course, different resistance laws lead to different governing equations (resistance forces may be proportional to the square of the velocity). Further, $a$ and $\alpha$ may depend on the flow (usually expressed as functions of the Reynolds number). In this study, $a$ and $\alpha$ are assumed constant for each time interval considered ("acceleration" and "collapse" of the bubble). If the evolution of $E_{p}$ with time is known, eqn (8) is linear. In the simplest theoretical case $E_{p}=$ constant (usually related to the vapor pressure), which leads to the solution:

$$
y_{2}=C_{1} e^{\theta_{1} t}+C_{2} e^{\theta_{2} t}+C_{3} e^{\theta_{3} t} .
$$

$\theta_{1}, \theta_{2}$, and $\theta_{3}$ are combinations of the constant coefficients of eqn (8b), and $C_{1}, C_{2}$, and $C_{3}$ depend on the boundary conditions of the problem. The roots of the third order characteristic equation obtained for eqn (8) may be 1) three real roots or 2) one real root and two complex roots. In the last case, the solution assumes the form:

$$
y_{2}=e^{\theta_{1} t}\left(C_{1} \cos \theta * *{ }_{1} t+C_{2} \sin \theta * *{ }_{1} t\right)+C_{3} e^{\theta_{3} t} .
$$

$\theta *_{1}$, and $\theta * *_{1}$ are the real and complex parts of the constants, respectively. This damped oscillatory behavior is expected, considering the physical arrangement shown in fig. 1(a). In this study the solution (9a) was tested with a two steps function for $E_{p}$, in the form given by fig. 1(b). $T_{a}$ is the time interval in which the bubble accelerates upwards. $T_{t}$ is the total time of the phenomenon. The real movement must consider the variation of $E_{p}$ (if $E_{p}$ varies). In the present study a procedure to adjust $C_{i}$ and $\theta_{i}$ of eqn (9a) proposed by Schulz [16], considering the nonlinearity of the equation, was applied, producing an adequate evolution of the amplitude $y_{2}$. $\theta_{i}$ may change because $a$ and $\alpha$ may assume different values for each time interval considered. 


\section{Experimental devices and procedures}

\subsection{Containers}

Two containers were tested. The first was built using a mercury lamp with the dimensions shown in fig. 2(a). The second was projected in order to better reproduce the hypothesis of the theoretical model. The horizontal movements of the fluid in the first container had negligible effect on the vertical movement of the container. The internal metal contacts could not be removed without opening the lamp and weakening too much its mechanical resistance.
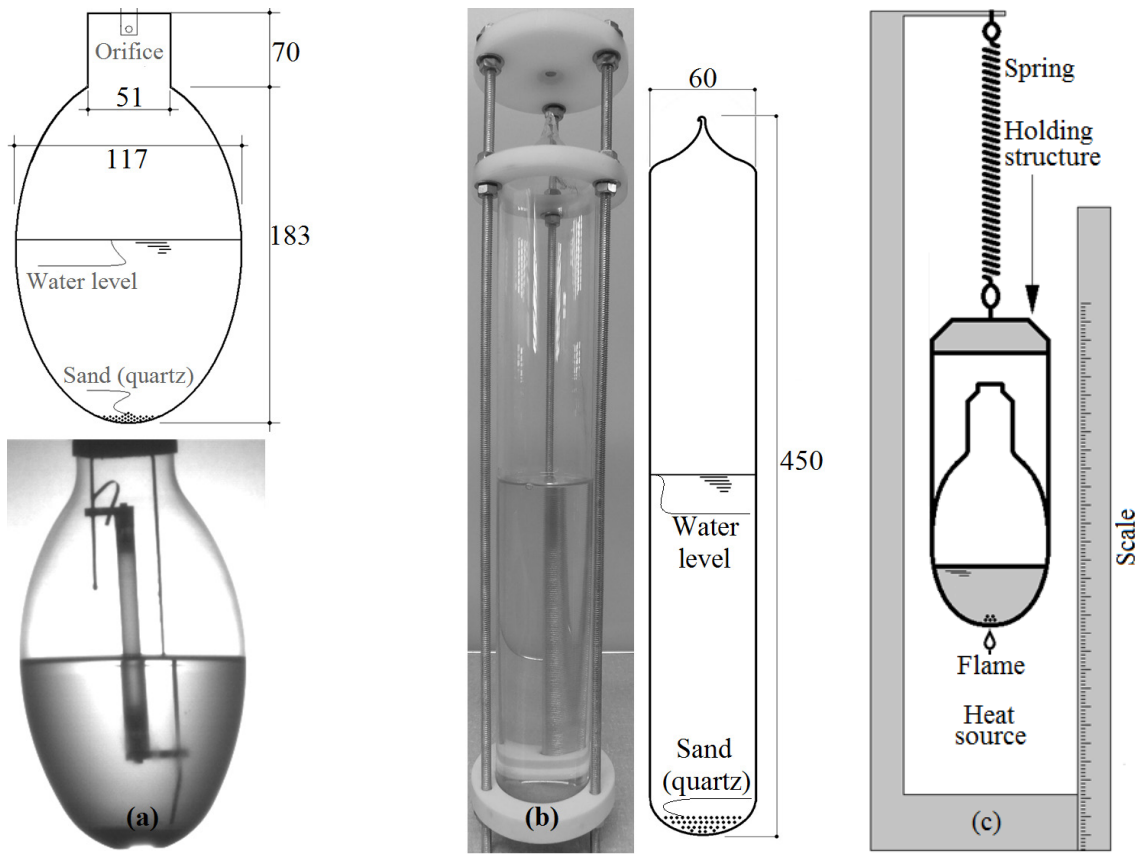

Figure 2: (a) Dimensions of the first container (mm); (b) Dimensions of the second container (mm); c) Experimental arrangement: container, holding structure, heat source (flame - ebullition events), spring (Adapted from Schulz et al. [15]).

The containers were totally filled with water. About $2 \mathrm{mg}$ of sand (quartz) was added to the water in each container, in order to avoid thermal convection at the bottom. The water was boiled during about 5 hours, allowing around $50 \%$ to evaporate through an orifice in the upper part, and producing an internal atmosphere mainly composed by water vapor. The orifice was closed by melting the glass while the water was boiling. The containers were then let to cool naturally. The internal pressure lowered until attaining the equilibrium condition. 
The masses used were: container 1, $157.8 \mathrm{~g}$; container 2, 370g; water 1, 680.4 g; water 2, 479.1g; spring and structure 1 (fig. 2(b)), 130.0 g. The constant of spring 1 was $K=19.92 \mathrm{~N} / \mathrm{m}$.

\subsection{Movement of the containers}

The results presented here are for the container of fig. 2(a), which first moved downwards reacting to the movement of the water in the inside, and then upwards due the spring. A very simple methodology allowed quantifying the movement: 1) it was recorded using a high velocity camera; 2) relevant frames were selected during slow motion analysis; 3) distances were measured digitally on these frames; 4) graphs showing the movement along time were plotted.

The scale sketched in fig. 2(c) allowed comparisons with the distances obtained digitally in the frames. Fig. 3 shows the measured amplitudes of the vertical movement of the first container for the four runs of this study. Large amplitudes were observed, with maxima in the range of 33 to $41 \mathrm{~mm}$, attained after about 25-28 ms. As mentioned, the diameter of the cavity was similar to the diameter of the container ( $\sim 17 \mathrm{~mm}$ horizontal and vertical).

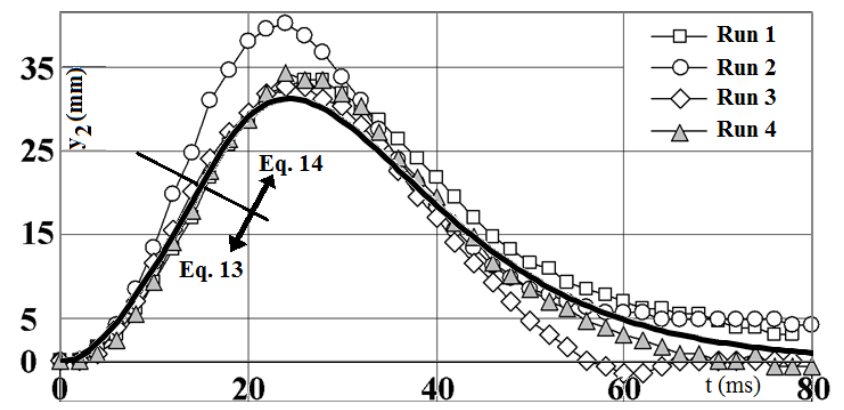

Figure 3: Amplitudes against time for the four runs ( $y_{2}$ of fig. 1(a)). The full line corresponds to predictions of eqns (13) and (14). The velocity of the camera was $500 \mathrm{fps}$.

\subsection{Evolution of the cavity}

The large size of the phenomena, recorded at $1250 \mathrm{fps}$, allowed observing details of the bubbles. The first four frames of fig. 4(a) shows the smooth increasing of the cavity during the growing period. The last three frames show the breakdown of the "ceiling" and a central macro scale jet "crashing" against the bottom. The central jet and the implosion of the bubble occur in the decreasing part of the graphs of fig. 3, being similar to the central microjets and the "torus like cavities" described for small bubbles by Shima [12]. The movement in the container of fig. 2(b) was registered at 320 fps. Fig. 4(b)-1 shows the system before the formation of the cavity, fig. 4(b)-2 shows a growing moment and fig. 4(b)-3 shows the inverted positions of water and vapour at maximum expansion. 


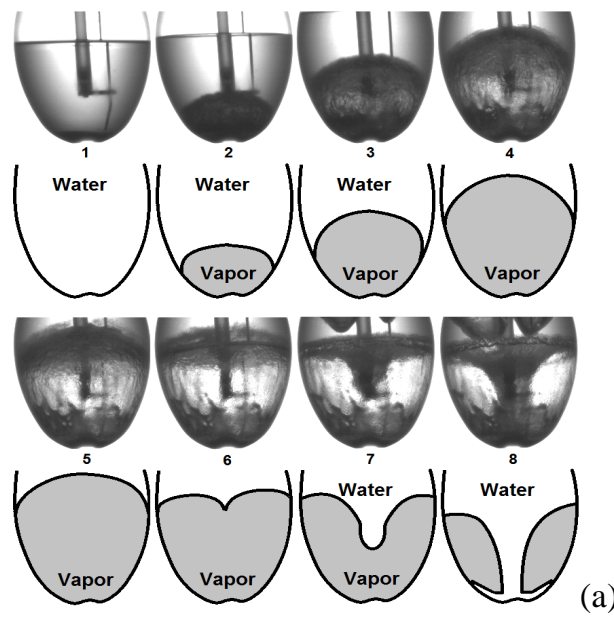

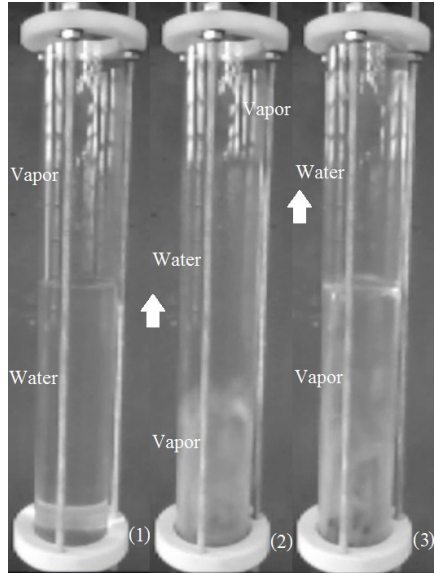

(b)

Figure 4: (a) Macro cavity growing and collapsing in the first container (1250 fps - each $5^{\text {th }}$ frame is reproduced); (b) Growing of cavity in the second container (320 fps).

\subsection{Forces on the container}

\subsubsection{Forces on the moving container}

Schulz et al. [15] obtained the force to move the device by multiplying $d^{2} y_{2} / d t^{2}$ by the mass (container+holding structure=287.8 g). $d^{2} y_{2} / d t^{2}$ was calculated as:

$$
\frac{d^{2} y_{2}}{d t^{2}}=\frac{y_{2}(t-2 \Delta t)+4 y_{2}(t-\Delta t)}{8 \Delta t^{2}}+\frac{-10 y_{2}(t)+4 y_{2}(t+\Delta t)+y_{2}(t+2 \Delta t)}{8 \Delta t^{2}} \text {. }
$$

Fig. 5 shows the calculated values and main trends. The cloud of points shows: 1) a maximum peak at $\sim 5 \mathrm{~ms}, 2$ ) a minimum peak at $\sim 25 \mathrm{~ms}$.

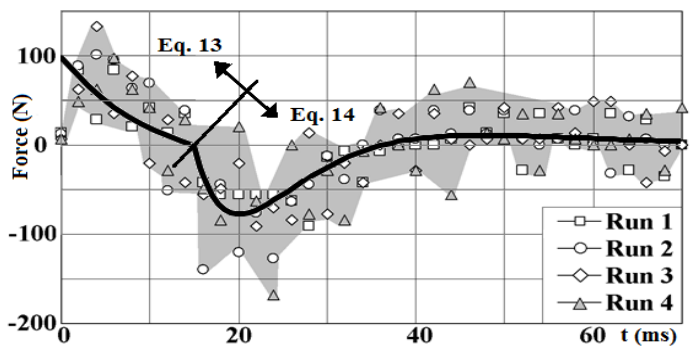

Figure 5: $\quad$ Observed force using eqn (10). Calculated force using eqns (13) and (14) (solid line).

\subsubsection{Forces on the fixed container}

In this case the container was fixed at a charge cell of $500 \mathrm{~N}$. The results are shown in fig. 6, and following characteristics were observed: 1) a maximum 
peak at $\sim 2.9 \mathrm{~ms}, 2$ ) a minimum peak at $\sim 31 \mathrm{~ms}, 3$ ) high frequency oscillations, probably reflecting the natural frequency of the charge cell.

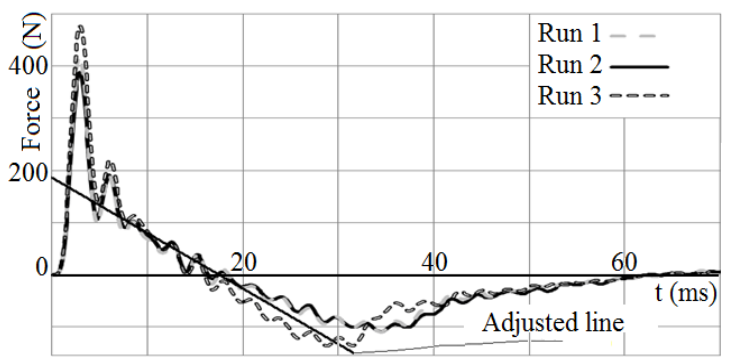

Figure 6: Evolution with time of the force applied on the container. Data obtained using a $500 \mathrm{~N}$ charge cell. Adapted from Schulz et al. [15].

The maximum measured peak of force was $476 \mathrm{~N}$, while the minimum peak was $-136 \mathrm{~N}$. The high frequency oscillations, if due to resonance of the charge cell, may imply in a real peak lower than the measured value. A straight line was adjusted for $10<t<20 \mathrm{~ms}$, furnishing $F=186.156-10651.6 t$, for visual comparisons between a linear behavior and the response of the charge cell. In fig. 6 the force is due to the acceleration of the mass of liquid (and not the container and the holding structure used in fig. 5).

\section{Evaluation $y_{2}$ using a two steps $E_{p}$ function}

Schulz et al. [15] used polynomial approximations to evaluate $E_{p}$ in eqn (8), based on the measured forces. Intending here to verify the answer of the equation to the simplest situations, the two steps situation sketched in fig. 1(b) was used. The two steps represent: 1) the acceleration of the liquid until the maximum ascending velocity, and 2) the deceleration of the liquid until the maximum amplitude was attained, followed by the collapse of the bubble (acceleration in the downward direction and implosion). Eqn (9a) is the solution, of both steps, but different coefficients may occur, considering variations in $a$ and $\alpha$. Schulz [16] considered the adjustment of nonlinear equations composed by sums of exponentials to known boundary conditions, procedures which were used here.

Following boundary conditions were used for the first step:

$$
\begin{array}{lll}
\left(i_{1}\right) y_{2}=0 \text { for } t=0 . & \left(i i_{1}\right) \frac{d y_{2}}{d t}=0 \text { for } t=0 . & \left(i i i_{1}\right) \frac{d^{2} y_{2}}{d t^{2}}=\frac{F_{0}}{m} \text { for } t=0 . \\
\left(i v_{1}\right) y_{2}=y_{2} \text { for } t=t_{A} . & \left(v_{1}\right) \frac{d y_{2}}{d t}=V_{A} \text { for } t=t_{A} . & \left(v i_{1}\right) \frac{d^{2} y_{2}}{d t^{2}}=0 \text { for } t=t_{A} .
\end{array}
$$

For step 2, the boundary conditions were initially adopted as 


$$
\begin{array}{lll}
\left(i_{2}\right) y_{2}=y_{A} \text { for } t=t_{A} . & \left(i i_{2}\right) \frac{d y_{2}}{d t}=V_{A} \text { for } t=t_{A} . & \left(i i_{2}\right) \frac{d^{2} y_{2}}{d t^{2}}=0 \text { for } t=t_{A} . \\
\left(i v_{2}\right) y_{2}=y_{P} \text { for } t=t_{P} . & \left(v_{2}\right) \frac{d y_{2}}{d t}=0 \text { for } t=t_{P} . \quad\left(v i_{2}\right) \frac{d^{2} y_{2}}{d t^{2}}=\frac{F_{P}}{m} \text { for } t=t_{P} .
\end{array}
$$

Having the form of the solution, the problem resumes in finding the constants $C_{1}, C_{2}, C_{3}, \theta_{1}, \theta_{2}, \theta_{3}$ of eqn (9a). For this study, $t_{A}=15 \mathrm{~ms}, t_{P}=25 \mathrm{~ms}$, $y_{A}=0,021 \mathrm{~m}, F_{0} / \mathrm{m}=340 \mathrm{~m} / \mathrm{s}^{2}$, and $y_{P}=0.033 \mathrm{~m}$ (lower observed value). For the first step, the coefficients of the arguments of the exponentials were obtained using the auxiliary function $S$ and the solution of $\theta_{3}$ shown below (see Schulz [16], for details)

$$
\begin{gathered}
S=-\theta_{1 \circ r 2}\left[y_{A} \exp \left(-\theta_{1 \text { or } 2} t_{A}\right)+\frac{V_{A}^{2}}{F_{0} / m}\right]-V_{A} \exp \left(-\theta_{1 \circ r 2} t_{A}\right) . \\
\theta_{3}=\frac{\ln \left[\frac{y_{A}}{F} \theta_{1} \theta_{2}-\frac{V_{A}}{F}\left(\theta_{1}+\theta_{2}\right)\right]}{t_{A}} .
\end{gathered}
$$

Eqn (11) furnished $\theta_{1}$ and $\theta_{2}$, obtained for a fixed value of $S$ (the same value for $\theta_{1}$ and $\theta_{2}$ ). Eqn (12) then furnished the value of $\theta_{3}$. The solution of $y_{2}$ obtained for the first step is given by:

$$
y_{2}=1.08024 \exp (-44.830 t)-0.73091 \exp (-57.095 t)+0.34933 \exp (-19,170 t) \text {. }
$$

For step 2, the last boundary condition (vi-2) was not used, because the coefficients of the arguments of the exponentials were chosen by trial using a computer program that stored the values which led to the lower differences between predicted and observed values of $y_{p}$ and of the amplitude at $t=60 \mathrm{~ms}$. The equation of $y_{2}$ obtained for the second step is given by

$$
y_{2}=0.57444 \exp (-110 t)+0.51945 \exp (-90 t)+0.075988 \exp (-190 t) .
$$

Eqns (13) and (14) were used to obtain the full line of fig. 3. Although no values of $a$ and $\alpha$ are known, so that the coefficients of eqn (8) are in fact unknown, it was shown that it is possible to adjust coefficients of solutions like eqn (9a) for the simplest behavior of $E_{p}$. Eqns (13) and (14) also allow calculating the acceleration of the container, so that also the force can be evaluated for both steps considered here. The solid lines of fig. 5 show the predicted forces, which present discontinuities in the derivatives along time, not expected in real situations. This fact points to the need for better knowledge of the evolution of $E_{\mathrm{p}}$, and the use of more representative resistance laws, in order to obtain a better predicted behavior. Notwithstanding the mentioned differences, the methodology was able to reproduce the general observed trends. 
The above discussion points to the convenience of the present formulation, given by eqn (7) in the more general way, in describing the behavior of the movement of the container, that is, the amplitude $y_{2}$. It was also shown that better knowledge of $E_{p}$, is necessary, and probably the application of more representative resistance laws (in which $a$ and $\alpha$ were defined) in order to obtain more realistic solutions for the force done to move the container.

The calculations are for the container of Figs 2(a) and 4(a). The cavity of fig. 4(b), having a maximum height of $\sim 22.0 \mathrm{~cm}$ and inverting the positions of water and vapor, is shown to attest the vertical one-dimensional movement. The volumes of the cavities in fig. 4(a) and 4(b) are $\sim 830 \mathrm{~cm}^{3}$ and $\sim 620 \mathrm{~cm}^{3}$, respectively.

\section{Conclusions}

This study shows the generation of movement of devices using macro-cavities in liquids. The involved forces allowed us to stretch a spring, showing that cavities generate "usable" work.

A general formulation to quantify the movement was described (eqn (7)). A simple situation of two pressure steps and resistance laws using the first power of the velocity was considered to predict observed movements.

The theoretical solution of this simplified case that reduces to a sum of three exponential functions was tested here, adjusting coefficients for both pressure steps. The adjusted results show that the general behaviour of the amplitudes and the involved forces may be obtained from this formulation, although the force predictions showed discontinuities in the derivatives along time, not observed in the mean behaviour of the measured data.

The results point to the adequacy of the formulation, reproducing well the evolution of the amplitude with time, and to the need of a better knowledge of the behavior of the excess of pressure $\left(E_{p}\right)$, in order to achieve better predictions of forces.

\section{Acknowledgements}

The authors thank CAPES, FAPESP, and CNPq, for the financial support.

\section{References}

[1] Falvey, H.T., Cavitation in chutes and spillways. 1.ed. Denver: United States Bureau of Reclamation, 145p, 1990.

[2] Sánchez, R., Juana, L., Laguna, F.V. and Rodriguez-Sinobas, L., Estimation of cavitation limits from local head loss coefficient. Journal of Fluids Engineering, October, Vol 130, 9p, 2008.

[3] d'Agostino, L. and Salvetti, M.V. (eds.). Fluid dynamics of cavitation and cavitating turbopumps. International Centre for Mechanical Sciences, CISM Courses and Lectures Vol 496, Springer Wien New York, 2007. 
[4] Kodama, T. and Tomita, Y., Cavitation bubble behavior and bubble-shock wave interaction near a gelatin surface as a study of in vivo bubble dynamics. Appl. Phys. B 70, pp. 139-149, 2000.

[5] Alehossein, H. and Qin, Z., Numerical analysis of Rayleigh-Plesset equation for cavitating jets. Int. Journal for Numerical Methods in engineering, Vol 72, pp. 780-807, 2007.

[6] Azuma, Y., Kato, H., Usami, R. and Fukushima, T., Bacterial sterilization using cavitating jet. Journal of Fluid Science and Technology, Vol. 2, N.1, pp. 270-281, 2007.

[7] Assis, M.P., Dalfré Filho, J.G., and Genovez, A.I.B., Cavitating jet devices for water disinfection (Equipamento tipo jato cavitante para a desinfecção de água - text in Portuguese). XVII Brazilian Symposium on Water Resources, 2008.

[8] Gaitan, D.F., Crum, L.A., Roy, R.A., and Church, C.C., Sonoluminescence and bubble dynamics for a single, stable, cavitation bubble. The Journal of the Acoustical Society of America, Vol. 91, N. 6, pp. 3166-3183, 1992.

[9] Brenner, M.P., Hilgenfeldt, S., and Detlef, L., Single bubble sonoluminescence. Reviews of Modern Physics (The American Physical Society), Vol. 74, N. 2, pp. 425-484, 2002.

[10] Wrbanek, J.D., Fralick, G.C., Wrbanek, S.Y. and Hall, N.C., Investigating sonoluminescence as a means of energy harvesting. In Millis, M.G. and Davies, E.W. (eds.) Frontiers of propulsional science, American Inst. of Aeronautics and Astronautics, Abstract NASA Technical Reports Server, pp. 605-637, 2009.

[11] Tullis, J.P., Hydraulics of pipelines - pumps, valves, cavitation, transients. $1^{\text {st }}$ edition, John Wiley and Sons. 266p, 1989.

[12] Shima, A., Studies on bubble dynamics. Shock Waves, Vol. 7, pp. 33-42, 1996.

[13] Leighton, T.G., Derivation of the Rayleigh-Plesset equation in terms of volume. ISVR Technical Report No. 308, University of Southampton, Institute of Sound \& Vibration Research, 17p, 2007.

[14] Tomita, Y., Tsubota, M. and An-naka, N., Energy Evaluation of shock wave emission and bubble generation by laser focusing in liquid nitrogen. International Symposium on Cavitation CAV 2001, session A2.006, 8p, 2001.

[15] Schulz, H.E., Lombardi, G., Nascimento, F.J., Trebi, H.J.D., Santos, J.N. dos and Simões, A.L.A., Usable work of macro-scale cavities in liquids. $J$. Braz. Soc. Mech. Sci. \& Eng., Rio de Janeiro, v. 34, n. 4, 2012.

[16] Schulz, H.E., Adjusting nonlinear equations with sums of exponentials: the possibility of inifinite solutions (Ajuste de equações não lineares com somas de exponenciais: a possibilidade de infinitas soluções - text in Portuguese). Laboratory of Rheology and Turbulence, School of Engineering at São Carlos, University of São Paulo, LTR Report 1/III/13, 2013.

[17] Bronson, R. and Costa, G., Differential Equations. Schaum's Outline Series, $3^{\text {rd }}$ Edition, McGraw Hill, 385p, 2006. 